\section{Cultura de publicación: realidades diferentes}

\section{Publication culture: two different realities}

Sr. Editor: El aumento en los estándares de investigación de las instituciones médicas en eventos científicos ha generado una conducta de publicación más competitiva entre profesionales médicos, en especial en los médicos jóvenes, en algunos países desarrollados como es el caso de China. Este comportamiento presenta puntos de vista diferentes en su apreciación. Se menciona que en China se da una prioridad un tanto irrazonable e innecesaria a los estándares de publicación de los médicos e incluso ha hecho que médicos jóvenes se sientan presionados para la publicación, lo cual consideran que afecta el interés hacia la adquisición de experiencia médica y desarrollo de habilidades interpersonales con el paciente en esta etapa de su formación. No obstante el perfil de producción científica es alto y con metas establecidas. ${ }^{1}$

Por otro lado, en países en vías de desarrollo, como en Perú, el perfil de publicación de profesionales médicos es bajo. Pereyra-Elias et al encontraron que en 11 años sólo el $8 \%$ de los trabajos presentados en los congresos de gastroenterología de Perú fueron publicados, a diferencia del congreso chileno de Gastroenterología con 25,1\% de publicaciones y muy distante a los congresos europeos con $60-70 \%$ de publicaciones ${ }^{2}$.

Se proponen diversos factores asociados a la pobre cultura de publicación médica en el Perú, como la falta de capacitación en redacción científica, factores económicos que limitan la generación de investigaciones de mayor nivel ${ }^{2,3}$. Sin embargo, en Perú no existen estrategias que incentiven la realización y publicación de investigaciones por parte de los profesionales médicos en los eventos científicos, o si las hay son escasas. Es poco probable el uso de Science Citation Index (SCI) cuando sólo se cuentan con dos revistas biomédicas pe- ruanas indexadas en Medline y ninguna en la Web of Knowledge.

Llama la atención que mientras en China se percibe presión para publicar, en Perú hay desinterés y una escasa presión para ello. Por tanto consideramos que en regiones como la nuestra, es necesaria la introducción de estándares de calidad en investigación y publicación en eventos científicos biomédicos y así fortalecer el desarrollo científico en cada área de la medicina, sin que ello desequilibre el interés e impacto de la formación médico profesional y académica.

Juan José Montenegro-Idrogo ${ }^{1,2}$, Yesenia M. Huerta-Collado ${ }^{1,2}$

${ }^{1}$ Facultad de Medicina, Universidad Nacional Mayor de San Marcos.

${ }^{2}$ Sociedad Científica San Fernando UNMSM (SCSF-UNMSM). Lima, Perú.

\section{Referencias}

1. Heng-Feng Yuan, Wei-Dong Xu, Hai-Yan Hu. The Young Chinese doctors and the pressure of publication. TheLancet 2013; 381: e4.

2. Pereyra-Elias R, Ng-Suen G, Luis Fernando, Toro-Polo LM, et al. Baja publicación de los trabajos presentados a los Congresos de la Sociedad de Gastroenterología del Perú 1998-2008. Rev Gastroenterol Perú 2011; 31 (2): 124-32.

3. Parra V, Monge E, Vildósola H. Factores relacionados con la producción científica de los médicos gastroenterólogos en Lima-Perú. Rev Gastroenterol Perú 2009; 29 (3): 226-33.

Correspondencia a:

Juan José Montenegro Idrogo

Arnaldo Marquez 579 Int 507. Lima 11, Perú.

Teléfono: (511) 961072186

montenegroidrogo@gmail.com 